\title{
Spectra and appearance of late gadolinium enhancement in magnetic resonance imaging of non-ischemic myocardial disease - from pattern to diagnosis
}

\author{
Christina Heilmaier ${ }^{1}$, Horst Franzen ${ }^{2}$, Kai Nassenstein ${ }^{2}$, Thomas W. Schlosser ${ }^{2}$, J örg \\ Barkhausen $^{3}$, Peter Hunold ${ }^{3}$
}

1. Department for Diagnostic Radiology, Stadtspital Triemli, Zurich, Switzerland. 2. Department for Diagnostic and Interventional Radiology and Neuroradiology, University Hospital Essen, Germany. 3. Clinic for Radiology and Nuclear Medicine, University Hospital Schleswig-Holstein, Campus Luebeck, Germany

Correspondence: Christina Heilmaier. Address: Department for Diagnostic Radiology Stadtspital Triemli, Birmensdorfer street 497, 8063 Zurich, Switzerland. Email: christina.heilmaier@gmx.de

Received: April 10, 2014

DOI : $10.5430 /$ ijdi.v1n2p118
Accepted: June 9, $2014 \quad$ Online Published: July 1, 2014

URL: http://dx.doi.org/10.5430/ijdi.v1n2p118

\section{Abstract}

Objective: Value of late gadolinium enhancement (LGE) in diagnosis of ischemic disorders in cardiac magnetic resonance is well-known. The purpose of the present paper was to describe more details and characteristics of LGE patterns arising in several non-ischemic myocardial diseases.

Methods: Cardiac MRI was performed in 1,905 patients at a 1.5-T MR system and showed LGE with 1,646/1,905 demonstrating LGE patterns or other diagnostic criteria (e.g. laboratory results, ECG, coronary angiography findings) regarded typical for ischemic heart disease. The remaining 259/1,905 patients revealed LGE related to non-ischemic myocardial disease. Two experts (radiologists and/or cardiologist) qualitatively described LGE pattern in consensus reading with regard to its site (left or right ventricle), extent (subendocardial, midmyocardial, subepicardial, transmural, pericardial), segment (according to the 17- segment model of left ventricle (LV)), distribution (streaky, patchy, punctual, spotty, crescent, diffuse), and intensity (weak, moderate, strong). Thereafter, results were correlated to final diagnosis based on synopsis of clinical features, laboratory results, ECG, biopsy, or other imaging procedures.

Results: In 250/259 patients (96.5\%) only the LV was affected by LGE, most frequently midmyocardial (166/259). In most cases this was due to peri-/myocarditis (especially if of weak-moderate intensity), while a weaker, streaky or spotty LGE basal or lateral pointed to HCM (39/166) and streaky and lateral to DCM (20/166). A midmyocardial, patchy LGE in segment 3 and 5 was caused by Fabry's disease (6x). In case of a transmural, spotty or patchy pattern LGE pointed to $\mathrm{HCM}$, if intensity was weak-moderate, or to peri-/myocarditis, when LGE was moderate-strong. In addition to that, subepicardial LGE in basal segments with patchy, streaky, or spotty distribution and moderate intensity was also due to peri-/myocarditis, while it was located midventricular, spotty and weak in TASH (transcoronary ablation of septum hypertrophy).

Conclusions: Several LGE patterns point to certain non-ischemic myocardial diseases and thereby might facilitate and confine differential diagnosis and might be useful in biopsy planing.

\section{Keywords}

Cardiac MRI, Late gadolinium enhancement, Pattern, Myocarditis 


\section{I ntroduction}

Within the last decade cardiac magnetic resonance imaging (CMR) has been established as a major diagnostic tool with great importance in clinical decision making. Due to robust technical features of the hardware and further software developments, the diagnostic potential and capabilities of CMR constantly broaden. Besides functional analysis and the assessment of the extent of viable and non-viable myocardium CMR has been increasingly appreciated in diagnosing and monitoring non-ischemic myocardial disease such as myocarditis or cardiomyopathy ${ }^{[1,2]}$ and is also known to play an important role with regard to prediction of adverse cardiac outcomes ${ }^{[3]}$.

Late gadolinium enhancement (LGE) is a substantial part of CMR and is highly valued in both differentiating between ischemic and non-ischemic myocardial disease as shown by various studies ${ }^{[1-11]}$ as well as identifying primary or secondary cardiomyopathy. LGE uses extracellular paramagnetic, Gadolinium-based contrast agents and reflects edema, myocyte necrosis or fibrosis of different etiologies. In healthy myocardial tissue the contrast agent usually equilibrates rapidly between the vascular space and interstitium; however, in injured myocardium, when fibrosis, disruption of cell membranes, or edema occur, the contrast agent increasingly gains accessibility to free water protons through penetration into the cell or pooling in the enlarged interstitial space which leads to a higher distribution volume and concentrations ${ }^{[4]}$. Concurrently, the wash-in and wash-out of the contrast agent can be slowed due to changes in coronary flow rates and capillary density. As a consequence, the contrast agent impounds, leading to T1-shortening, thereby augmenting signal intensity on T1-weighted images relative to that of the normal myocardium.

While LGE in myocardial infarction is situated either subendocardial or transmural and follows a coronary artery distribution ${ }^{[2]}$, there are different distribution patterns in non-ischemic disorders. Non-ischemic LGE can be found in the left and right ventricle, can show a diffuse or focal spreading independent of a vascular distribution, and can have different signal intensity. Thus, the characterization of LGE pattern might facilitate and confine differential diagnosis and might serve as a guide for endomyocardial biopsy to improve diagnostic yield ${ }^{[1]}$.

The purpose of this study was to identify and index typical LGE patterns and characteristics of various non-ischemic etiologies in order to correlate them with different non-infarction-related myocardial disorders (e.g. peri-/myocarditis, dilated cardiomyopathy, hypertrophic obstructive cardiomyopathy). To our knowledge, our study is the first to pursue this question both in a systematic approach describing the site (left/right ventricle), preferred segment, distribution (e.g. patchy, spotty), and intensity of LGE and in a large study cohort of 259 patients.

\section{Methods}

This retrospective study was approved by the local ethics committee and was performed in keeping with the declaration of Helsinki.

We cross-referenced our institutional imaging and report database to identify all patients, who underwent contrastenhanced cardiac magnetic resonance imaging (CMR) within a 50-months period for different clinical indications. In patients with severe arrhythmias scans were aborted before contrast media was applied and data was excluded from analysis. Complete contrast-enhanced CMR data sets acquired within the given time frame were available from 5,676 subjects. In 1,905/5,676 (34\%) late gadolinium enhancement (LGE) was detected that was related to ischemic damage in $1,646 / 1,905$ subjects (86\%) and due to non-ischemic disease in 259/1,905 individuals (14\%). Ischemic damage was regarded to be the sequelae of coronary artery disease and myocardial infarction with subjects being assigned to this patient group if: (a) there was a stenosis $\geqslant 70 \%$ in at least one coronary artery in coronary angiography (LGE was correlated with the vessel territory that displayed a stenosis in coronary angiography) (1,121 subjects), (b) patients had a history of myocardial infarction (MI) and signs of MI in ECG, echocardiography, and/or nuclear imaging, and corresponding LGE patterns in CMR (201 subjects), (c) it was established that the subject suffered from chronic MI 
(thinner wall in cine sequences and akinesia) (82 subjects), or (d) LGE-CMR revealed a typical chronic infarction LGE (40 subjects) as described by others ${ }^{[2]}$. Moreover, patients who displayed a mixed ischemic and non-ischemic LGE pattern were excluded from further analysis (202 patients). As a consequence 259 patients (male/female: 179/80; age 53.2 \pm 10.9 years) formed the focus of this study on non-coronary related LGE and underwent CMR examinations 1.6 days after initial symptoms on average (range, 0-5 days). Table 1 provides an overview of the indications for patients' referral to CMR examination. Suspected peri-/myocarditis means that the patient is believed to suffer from peri-/myocarditis due to clinical symptoms, laboratory results or other imaging modalities and CMR is performed to confirm the suspected diagnosis. Control on the other hand was done to look for remaining disease activity after the initial diagnosis was made and treatment was started. "Cardiac intervention" encompasses procedures such as coronary angiography (most cases) or ablation in atrial fibrillation.

Table 1. Summary of clinical indications to perform cardiac MRI in the study group.

\begin{tabular}{ll}
\hline Reasons for referral to CMR & No. of patients \\
\hline suspected or control after peri-/myocarditis* & $143(55 \%)$ \\
suspected cardiomyopathy & $55(21 \%)$ \\
control after TASH & $16(6 \%)$ \\
suspected sarcoidosis & $7(3 \%)$ \\
suspected ARVD & $6(2 \%)$ \\
suspected amyloidosis & $5(2 \%)$ \\
suspected Fabry's disease & $2(1 \%)$ \\
other diseases & $25(10 \%)$ \\
\hline$*$ In our study "suspected" always means that there is clinical hint for a certain disease, which now should be confirmed by CMR (first CMR examination). Contrary to that "control" indicates that the \\
patient was diagnosed to suffer from e.g. myocarditis before and now undergoes repetitive CMR for evaluation of course of disease. \\
ARVD = arrhythmogenic right ventricular dysplasia, HOCM = hypertrophic obstructive cardiomyopathy, TASH = transcoronary ablation of septum hypertrophy
\end{tabular}

\section{MR imaging}

MR examinations were performed on different 1.5T scanners (MAGNETOM Avanto or Sonata; Siemens AG Healthcare Sector, Erlangen, Germany) in two affiliated institutions. In all patients the uniformly utilized CMR protocol included an ECG-triggered breath-hold segmented steady-state free precession (SSFP) cine sequence ("trueFISP"; TR, 3.0msec; TE, $1.5 \mathrm{msec}$; flip angle, $60^{\circ}$ ) with a slice thickness of $8 \mathrm{~mm}$. After acquisition of long-axis views (two-, three- and four-chamber view), contiguous short-axis slices were obtained covering the entire left ventricle (LV) from base to apex without interslice gap. In particular patients depending on indication (e.g. suspected myocarditis), T2-weighted turbo spin-echo (TSE) sequences (TR, two R-R intervals; TE, 104msec) were collected in selected orientations to screen for the presence of myocardial edema. However, this sequence was not part of data analysis.

Contrast-enhanced images were acquired to assess the existence of myocardial LGE. For this purpose an extracellular contrast agent, either gadopentetate dimeglumine (Magnevist ${ }^{\circledR}$; BayerSchering AG, Berlin, Germany) or gadodiamide (OmniscanTM; GE Healthcare Bio-Sciences, Amersham, Great Britain), was injected in a concentration of $0.2 \mathrm{mmol} / \mathrm{kg}$ body weight and images were acquired between 8 and $15 \mathrm{~min}$ after administration of the contrast agent using a breath-hold ECG-triggered 2D inversion-recovery TurboFLASH sequence (TR/TE, $8 / 4 \mathrm{~ms}$; flip angle, $25^{\circ}$; slice thickness, $8 \mathrm{~mm}$ ). In each patient the inversion time (TI; nonselective inversion pulse) was manually adjusted between 200-260 ms to null the signal of normal myocardium and enhance the conspicuity of abnormal tissue. Images were acquired in three long-axis 
views and all contiguous short-axis slices with a typical in-plane spatial resolution of $1.6 \mathrm{~mm} \times 1.3 \mathrm{~mm}^{2}$ (depending on the field of view).

\section{I mage analysis}

As mentioned before, further analysis was only performed in patients with LGE caused by non-infarction-related disorders. Thus, data of 259 individuals with non-ischemic disease were reviewed in random order and retrospectively evaluated in a consensus reading by two radiologists (C.H., P.H.) and/or cardiologists (H.F., O.B.) experienced in CMR (6, 11,3 , and 9 years of experience, respectively). Readers were asked to qualitatively describe the appearance of the LGE following the approach as given in Figure 1. At first, the site of LGE (left ventricle, right ventricle, both) and then the extent (subendocardial, midmyocardial, subepicardial, transmural, pericardial) of LGE were analyzed. Thereafter, the affected segments of the LV were assessed using the 17-segment model of the LV of the American Heart Association ${ }^{[12]}$. The forth step included description of LGE distribution: punctual (= small points of LGE, 1-2mm), spotty (= rounded lesions of intermediate size, $3-5 \mathrm{~mm}$ ), patchy (= rounded or ovular lesions of larger size, $>5 \mathrm{~mm}$ ), crescent, diffuse $(=$ mixture of rounded and streaky patterns), streaky (= longish lesions of different sizes). In a last step signal intensity was quantatively evaluated within the LGE area of most uniformly appearing pattern and was categorized as follows: weak intensity $>$ mean +1 standard deviation (SD) of normal myocardium; moderate intensity $>$ mean +2 SD; strong intensity $>$ mean $+3 \mathrm{SD}$. The readers had no access to other sequences of the MRI studies apart from the LGE imaging (especially not to T2-weighted images) and were blinded to all clinical information.

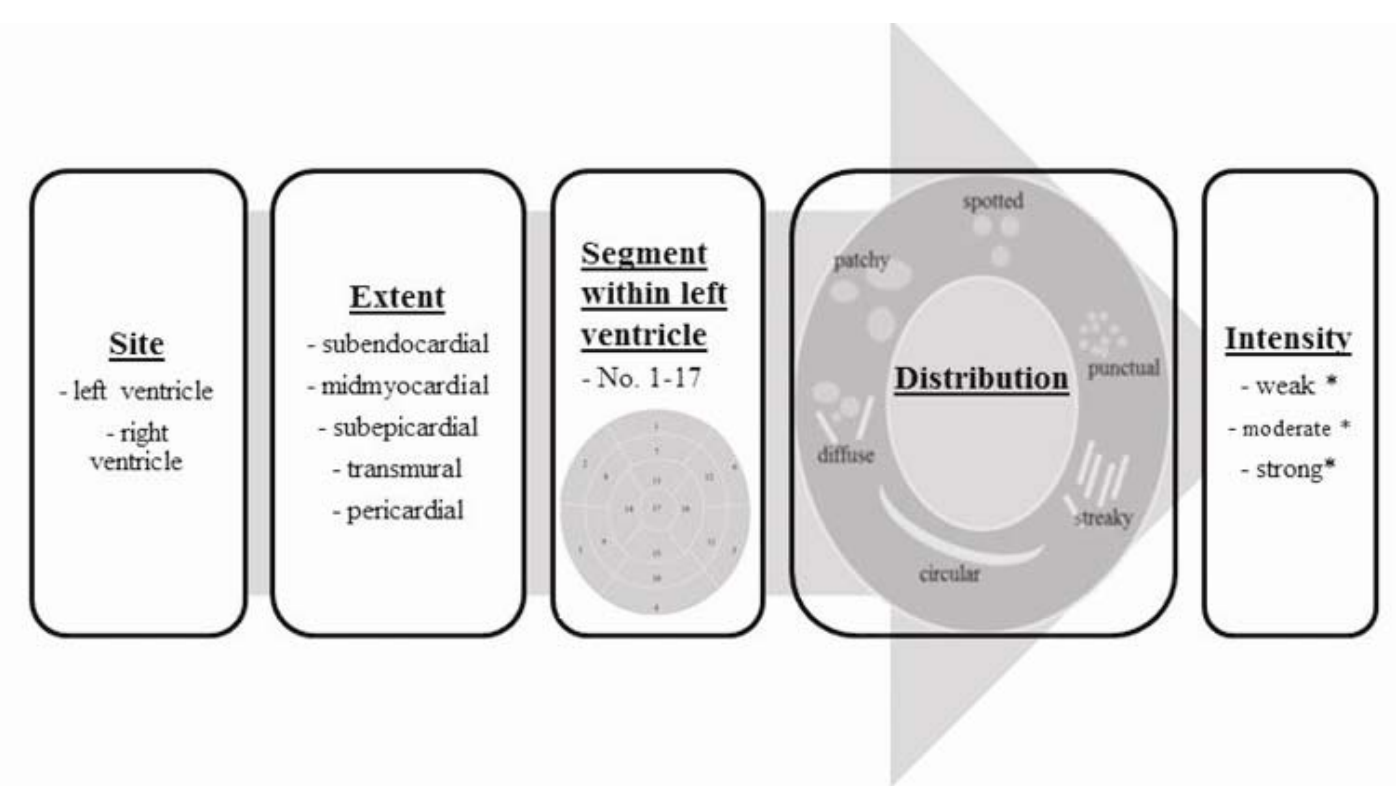

Figure 1. Systematic analysis of the LGE pattern.

Afterwards, results of LGE analysis were correlated to the underlying pathophysiologic process, which was established by final diagnosis based on a synopsis of clinical features, laboratory results, ECG, biopsy, or other imaging procedures such as echocardiography or nuclear imaging. For this purpose, diagnostic criteria as provided by Table 2 were applied.

\section{Statistics}

For the quantitative analysis results are provided as means \pm standard deviation. The $t$-test was applied to look for differences (e.g. of signal intensity of LGE patterns in myocarditis versus hypertrophic cardiomyopathy) with a $p$-value $<$ 0.05 defining statistical significance. All calculations were done using commercially available software (SPSS, release 20.0; IBM SPSS, Chicago, IL, USA). 
Table 2. Survey on final diagnoses as present in patient cohort based on a synopsis of clinical features, laboratory results, ECG, biopsy, or other imaging procedures such as echocardiography or nuclear imaging.

\begin{tabular}{|c|c|c|}
\hline Diagnosis & Abstract of diagnostic criteria & $\begin{array}{l}\text { No. of } \\
\text { patients }\end{array}$ \\
\hline Myocarditis & $\begin{array}{l}\text { MB creatine-kinase } \uparrow, \text { Troponin I and } \mathrm{T} \uparrow, \mathrm{CRP} \uparrow, \mathrm{ESR} \uparrow, \mathrm{ECG} \text { abnormalities (e.g. tachycardia, } \\
\text { extrasystoles), LV systolic dysfunction in echocardiography, biopsy }\end{array}$ & $\begin{array}{l}112 \\
(43 \%)\end{array}$ \\
\hline $\mathrm{H}(\mathrm{O}) \mathrm{CM}$ & $\begin{array}{l}\text { dyspnea, ECG changes (e.g. abnormal Q waves, short PR interval, delta waves), asymmetric } \\
\text { septal hypertrophy or SAM phenomenon in echocardiography, biopsy }\end{array}$ & $54(21 \%)$ \\
\hline cardiac intervention & exclusion of other etiologies, clinical history & $23(9 \%)$ \\
\hline DCM & $\begin{array}{l}\text { signs of LV dysfunction, cardiac arrhythmia, dilatation of both ventricles in echocardiography } \\
\text { and hypokinesia, exclusion of ischemic cardiomyopathy in coronary angiography }\end{array}$ & $22(8 \%)$ \\
\hline peri-myocarditis & same as in myocarditis + pericardial effusion in echocardiography & $13(5 \%)$ \\
\hline Fabry's disease & $\begin{array}{l}\text { clinical history, acroparesthesia or dermatological manifestations, level of alpha-galactosidase } \\
\text { activity in blood, chromosomal analysis, cardiac biopsy }\end{array}$ & $7(3 \%)$ \\
\hline fibrosis unknown origin & exclusion of other etiologies, biopsy & $6(2 \%)$ \\
\hline sarcoidosis & $\begin{array}{l}\text { serum ACE level } \uparrow \text {, in echocardiography dilated ventricle with wall motion abnormalities or } \\
\text { wall thinning in the ventricular septum, biopsy }\end{array}$ & $5(2 \%)$ \\
\hline scleroderma & $\begin{array}{l}\text { antinuclear antibodies } \uparrow, \text { ECG abnormalities such as elevated RVSP, pericardial effusion, } \\
\text { increased RV dimension, and left atrial enlargement, biopsy }\end{array}$ & $4(2 \%)$ \\
\hline pericarditis & $\begin{array}{l}\text { pericardial rub and new S3 sound on auscultation, convexly elevated J-ST segment in ECG, } \\
\text { inflammatory blood markers } \uparrow \text {, pericardial effusion in echocardiography, biopsy }\end{array}$ & $3(1 \%)$ \\
\hline ARVD & $\begin{array}{l}\text { ventricular arrhythmias, palpitations, signs of heart failure, epsilon wave in ECG, } \\
\text { hypo-/dyskinesia of RV in echocardiography, biopsy }\end{array}$ & $2(1 \%)$ \\
\hline amyloidosis & $\begin{array}{l}\text { ECG changes (low voltage with abnormal axis and poor R-wave progression in precordial } \\
\text { leads), LV thickening and signs of diagnostic dysfunction in echocardiography, biopsy }\end{array}$ & $2(1 \%)$ \\
\hline Churg-Strauss disease & $\begin{array}{l}\text { blood eosinophilia and inflammatory syndrome, antineutrophil cytoplasmic antibodies } \uparrow, \text { ECG } \\
\text { abnormalities (high-degree atrioventricular dissociation, left or right bundle-branch block, } \\
\text { indeterminate conduction delay, LV hypertrophy with ST-T abnormalities, and presence of } \\
\text { arrhythmias), in echocardiography wall motion abnormalities, pulmonary hypertension and/or } \\
\text { obliterated ventricles; biopsy }\end{array}$ & $2(1 \%)$ \\
\hline neoplasia & exclusion of other etiologies, biopsy & $2(1 \%)$ \\
\hline $\begin{array}{l}\text { Wegener's } \\
\text { granulomatosis }\end{array}$ & $\begin{array}{l}\text { inflammatory markers } \uparrow \text {, cytoplasmic pattern antineutrophil cytoplasmic antibodies with } \\
\text { antigen specificity for proteinase } 3 \text {, nephritic urinary sediment, vasculitis, ECG changes } \\
\text { (arrhythmias), pericardial effusion in echocardiography, biopsy }\end{array}$ & $1(0.4 \%)$ \\
\hline $\begin{array}{l}\text { changes after } \\
\text { radiotherapy }\end{array}$ & exclusion of other etiologies, clinical history & $1(0.4 \%)$ \\
\hline
\end{tabular}

\section{Results}

All CMR examinations were of adequate quality and well tolerated by the patients; therefore, data of 259 subjects with non-ischemic myocardial disorder were available and retrospectively evaluated. 


\section{Final diagnoses}

Of these patients 163/259 (63\%) underwent cardiac biopsy after CMR examination, revealing the following diagnoses: 75 peri-/myocarditis, $43 \mathrm{H}(\mathrm{O}) \mathrm{CM}, 22 \mathrm{DCM}, 6$ Fabry's disease, 6 fibrosis unknown origin, 2 scleroderma, 2 sacoidosis, 2 Churg-Strauss disease, 2 neoplasia, 1 arrhythmogenic right ventricular dysplasia (ARVD), 1 amyloidosis, and 1 Wegener's granulomatosis. In all other patients diagnoses were based on a synopsis of clinical examination results, laboratory values and/or other imaging modalities. Table 2 provides a summary of patients' diagnoses.

\section{LGE site and segment}

LGE was noticed within the left ventricular (LV) myocardium in 250/259 (96.5\%) subjects and within the right ventricle (RV) in 5/259 (2\%) individuals of our study group, 4 (1.5\%) patients had LGE in both ventricles. Isolated LGE in the RV was caused by either Churg-Strauss disease, ARVD or neoplasia, but did not display specific patterns. All patients with LGE located both in the LV and RV also had pericardial enhancement and suffered from peri-/myocarditis. In these cases LGE was mainly transmural with a patchy distribution.

Figure 2 gives a "bull's eye" diagram that in accordance with the 17-segment model of the LV of the American Heart Association ${ }^{[12]}$ illustrates the localization of the LGE within the LV: the darker the color in the diagram, the more often the respective segment was involved by LGE. As can be drawn from Figure 2, especially the lateral wall of the LV (segment 5 , 6,11 , and 12) as well as the inferoseptal segment frequently had LGE, with a relative uniform affection of the other segments. In 32/259 (12\%) subjects LGE occurred in all LV segments.

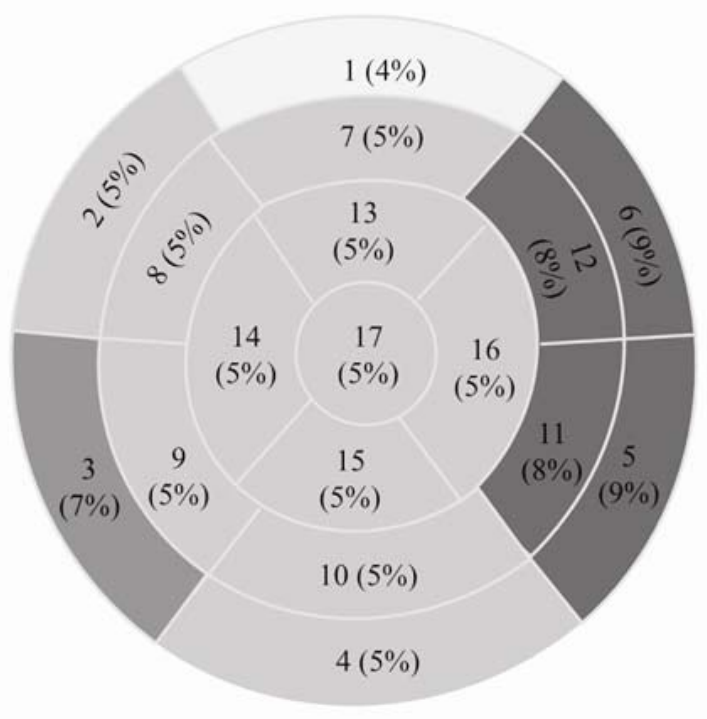

Figure 2. "Bull's Eye" Diagram (according to the 17-Segment Model of the LV of the American Heart Association (12)). The figure shows the frequency of involvement of the different segments (given by their numbers): the darker the respective segment appears, the more often LGE was situated in this segment.

NB: Numbers in parentheses are percentages relative to the total number of involved segments.

\section{Signal intensity}

Signal intensity (see Figure 3) was mainly weak or moderate; a strong intensity occurred considerably less $(p<0.05)$ and was only measured in $8 / 259$ patients (3\%). 


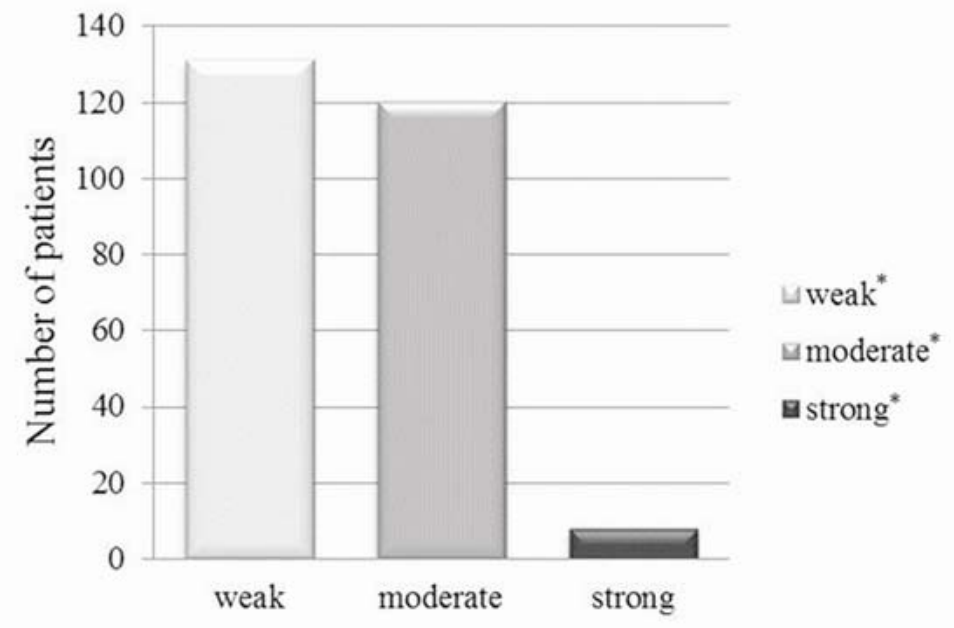

Figure 3. Signal intensity of the LGE. As demonstrated by the diagram in most patients a weak or moderate signal intensity of the LGE was present.

* Weak intensity $>$ mean +1 standard deviation $(\mathrm{SD})$; moderate intensity $>$ mean $+2 \mathrm{SD}$; strong intensity $>$ mean $+3 \mathrm{SD}$

\section{From pattern to diagnosis}

When the different LGE characteristics were fused and read in a synopsis instead of single parameters, some patterns pointed to certain non-ischemic cardiac disorders as listed in Table 3:

Table 3. Synopsis of patterns arising during different non-ischemic cardiac diseases.

\begin{tabular}{lllll}
\hline Disease & Extent & Location & Distribution & Intensity \\
\hline peri-/myocarditis & transmural & no preference & patchy>spotty & moderate-strong \\
& midmyocardial & no preference & patchy>streaky $>$ spotty & weak-moderate \\
& subepicardial & basal & patchy>streaky>spotty & moderate \\
& transmural & no preference & patchy >spotty & weak-moderate \\
$\mathrm{H}(\mathrm{O}) \mathrm{CM}$ & midmyocardial & basal, lateral & streaky>spotty>patchy & weak \\
& midmyocardial & diffuse & punctual & weak \\
TASH & subepicardial & midventricular & spotty & weak \\
& midmyocardial & diffuse & streaky & weak \\
DCM & midmyocardial & segment 3 and 5 & patchy & weak \\
Fabry's disease & & &
\end{tabular}

$\mathrm{DCM}=$ dilated cardiomyopathy, $\mathrm{H}(\mathrm{O}) \mathrm{CM}=$ hypertrophic (obstructive) cardiomyopathy, TASH = transcoronary ablation of septum hypertrophy

- In case of a transmural LGE pattern (37 subjects) two diseases were present: peri-/myocarditis (23 subjects) and hypertrophic cardiomyopathy (HCM; 14 subjects). In both diseases no segment preference was detected and the distribution was either spotty (12 patients, 8 peri-/myocarditis, $4 \mathrm{HCM}$ ) or patchy ( 25 patients, 15 peri-/myocarditis, 10 $\mathrm{HCM})$. However, in patients with peri-/myocarditis signal intensity was considerably stronger (14x moderate, $7 \mathrm{x}$ weak, $2 \mathrm{x}$ strong) than in those with HCM (10x weak, $4 \mathrm{x}$ moderate $)(p<0.05)$. 
- A subepicardial LGE extent (40 patients) with a patchy (17x), streaky (11x) or spotty (6x) distribution and a mainly moderate signal intensity (27x moderate, $5 \mathrm{x}$ weak, $2 \mathrm{x}$ strong) was again often seen in per-/myocarditis ( 34 subjects), this time preferably located in the basal segments (see Figure 4). Apart from peri-/myocarditis a subepicardial LGE was detected after TASH (tanscoronary ablation of septum hypertrophy; 6 subjects), when the distribution was spotty, the signal intensity weak and the affected segment midventricular. Differences in signal intensity were significant $(p<0.05)$ when compared to peri-/myocarditis. TASH is usually performed in patients with severe hypertrophic obstructive cardiomyopathy (HOCM). During this interventional procedure an infarct is set through occlusion of the first septal branch of the left anterior descending artery, consequently leaving septal scars ${ }^{[19]}$.

- A less clear pattern was evident with midmyocardial LGE, which was the most popular extent (166 patients). Frequent distributions were patchy $(75 x)$, streaky $(57 x)$ or spotty $(25 x)$, while punctual $(8 x)$ and crescent $(1 x)$ were only seldom detected. Again, peri-/myocarditis was the most popular diagnosis $(92 \mathrm{x})$. A patchy $(61 \mathrm{x})$, or less frequently streaky $(17 \mathrm{x})$ or spotty $(14 \mathrm{x})$, LGE with weak to moderate signal intensity and without segment preference indicated peri-/myocarditis. A midmyocardial LGE, preferably seen in the basal and lateral segments, with a streaky (20x), patchy (8x) or spotty (11x) distribution and a weak signal intensity was diagnosed as $\mathrm{H}(\mathrm{O}) \mathrm{CM}$ in 39 subjects. Contrary to that a midmyocardial LGE with diffuse affection of the LV, a streaky distribution and a weak signal intensity was described in dilated cardiomyopathy (DCM; 20x) (see Figure 5). Both cardiomyopathies had significantly lower signal intensity than peri-/myocarditis $(p<0.05)$. Based on the affected segments (No. 3 and 5) LGE pattern seen in Fabry's disease $(6 \mathrm{x})$ differed from LGE detected in DCM and H(O)CM. The LGE in Fabry's disease was mainly patchy and of weak signal intensity. And finally, a midmyocardial LGE was found after TASH (9x), when it was punctual (8x), of weak intensity and spread around the whole LV.

- Twenty subjects had a subendocardial LGE described as being patchy (11x), spotty (5x), streaky (3x) or crescent (1x) in appearance, but correlation with a specific non-ischemic cardiac disorder failed and/or the number of underlying disorders (e.g. Churg-Strauss disease, 2x; amyloidosis, $2 \mathrm{x}$ ) was too low to determine a characteristic pattern

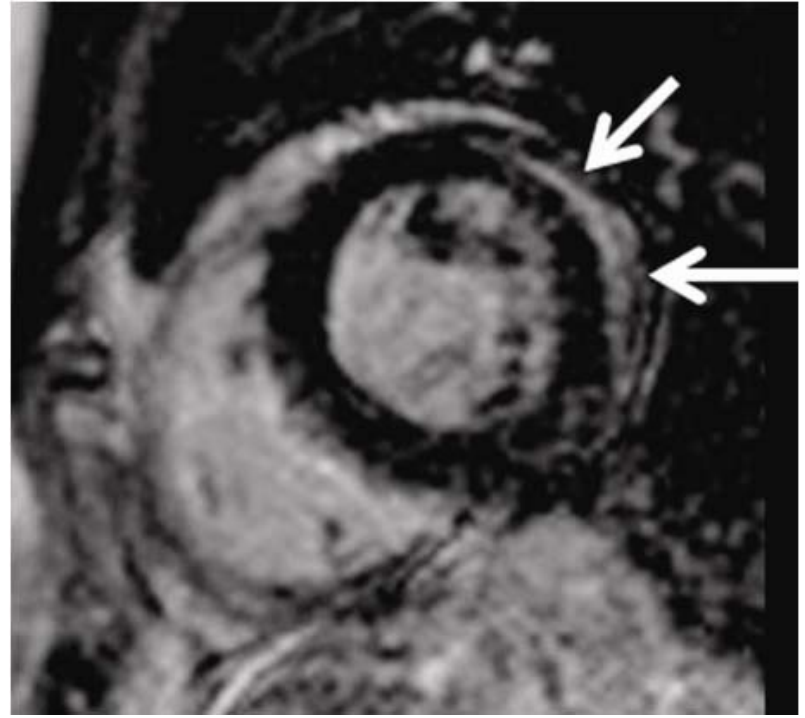

Figure 4. LGE in myocarditis. 25 year-old female patient suffering from myocarditis proven by biopsy. The short axis view of the LGE showed a subepicardial, streaky and crescent enhancement (arrow) in the lateral segments which confined differential diagnosis to myocarditis.

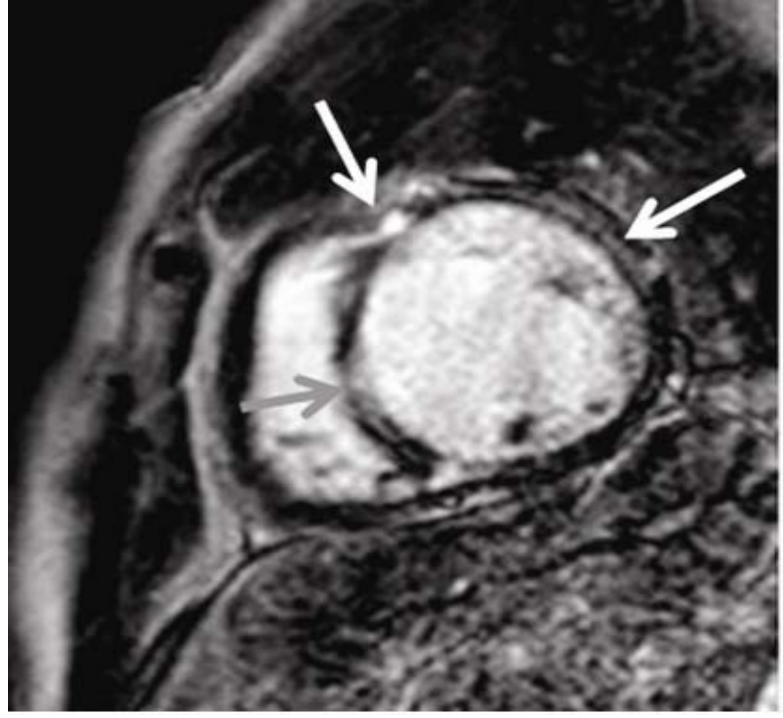

Figure 5. LGE in DCM. LGE in the septum (subendocardial, grey arrow), in the lateral wall (midmyocardial, white arrow) as well as in the anterior wall at the insertion of the right ventricle (transmural, white arrow) in a 47 year-old male patient with dilated cardiomyopathy (DCM). 


\section{Discussion}

"From pattern to diagnosis" - in performing this descriptive study we aimed to learn more about different patterns of late gadolinium enhancement (LGE) possibly linked to certain non-ischemic myocardial diseases, which might facilitate and confine differential diagnosis considerations.

The implementation of LGE CMR imaging enabled the differentiation between viable and non-viable myocardium ${ }^{[13,14]}$ and was at first utilized for identification and characterization of ischemic heart diseases. The characteristic pattern in these patients always involves the subendocardial layer, but may cover the whole myocardial wall, and is typically confined to a vascular territory ${ }^{[2,13]}$. Not only the LGE pattern itself might be beneficial in clinical practice but also its intensity as demonstrated by Stork et al. ${ }^{[11]}$, who described a correspondence of LGE intensity with cardiac function parameters as well as with the frequency of cardiac events; thus, LGE can be regarded as useful parameter for risk stratification $^{[15]}$.

Within the last years more and more studies were conducted using the "from diagnosis to pattern" way, which is the contrary approach compared to ours. Through these studies some patterns were identified that were related to certain non-ischemic disorders ${ }^{[1-5]}$. The aim of our study was to provide a structured approach in LGE analysis, which might be helpful in confining and arriving at the correct diagnosis, enabling appropriate treatment of non-ischemic diseases, thereby possibly reducing morbidity and mortality.

When assessing the enhancement patterns we first concentrated on the location within the LV or RV wall and its distribution, before we assigned the LGE to the segments involved and the signal intensity. Afterwards, all findings were analyzed in context and linked to the underlying diseases.

Due to its appearance in various diseases analysis of midmyocardial LGE was most challenging in our study with most patients suffering from peri-/myocarditis, in which LGE distribution was variable encompassing streaky, patchy or spotty patterns. This more diffuse damage than found in myocardial infarction is traced back to islands of necrotic cells scattered throughout disease foci. In contrast to HCM and DCM, the main differential diagnoses to peri-/myocarditis in midmyocardial LGE, signal intensity was stronger in peri-/myocarditis. In addition to that preferred LGE localizations (basal and lateral) pointed to HCM and DCM, while peri-/myocarditis was spread throughout the LV without a favored localization. In general, especially moderate-strong signal intensity seemed to be the most sensitive indicator for diagnosis of peri-/myocarditis when compared to all other non-ischemic diseases.

In our study a streaky midmyocardial LGE situated in the lateral LV segments pointed to DCM, which was identical to what was reported in other studies ${ }^{[16]}$. Contrary to that septal involvement was hardly seen in our study ${ }^{[1,16]}$, which might reflect different underling etiologies of DCM (e.g. alcohol, drug intoxication). Compared to peri-/myocarditis signal intensity was considerably weaker in DCM; thus, for us the combination of streaky, weak LGE in midmyocardium was most indicative of DCM.

Interestingly, a midmyocardial streaky or spotty LGE localized basal or lateral was linked to HCM in our study and no preferred septal involvement was seen, which was reported by others before ${ }^{[1,11,17,18]}$. Again, differentiation from peri-/myocarditis was most sensitive by signal intensity, which was considerably weaker in HCM. Despite being a rarity, it is important to diagnose a disease with a midmyocardial, patchy LGE of weak signal intensity localized in segment 3 and 5 of the LV: Fabry's disease. This X-linked genetic disorder causes an idiopathic LV hypertrophy ${ }^{[19]}$. While the non-subendocardial location of the enhancement limited to the basal infero-lateral wall (segment 5) was well known before ${ }^{[19]}$, involvement of the basal infero-septal segment (segment 3) had not been described so far. Differentiation from peri-/myocarditis was almost impossible in some cases, but a more confined affection of the LV and weaker signal intensity pointed to Fabry's disease, while LGE in peri-/myocarditis was often more diffuse affecting more than 2 segments and of stronger signal intensity. 
In accordance with other studies subepicardial LGE was almost exclusively found in patients with peri-/myocarditis ${ }^{[1,11]}$, while sarcoidosis another known disease with subepicardial enhancement and a cardiac involvement of $50 \%{ }^{[20]}$ did not show a specific pattern in our study ${ }^{[8,11]}$. However, as only 2 patients suffered from sarcoidosis in our study a detailed analysis of LGE pattern was not possible.

As mentioned before, subendocardial LGE is mainly due to myocardial infarction, albeit different studies reported about non-vascular causes such as amyloidosis ${ }^{[7]}$, Churg-Strauss disease or cardiac transplant; however, the number of affected patients was very limited in our study population (amyloidosis, 2x; Churg-Strauss disease, 2x), which (1) prevents a more detailed analysis and (2) might be the explanation why we did not detect a specific subendocardial pattern pointing to a distinct disorder.

We are aware of several limitations of our study: first, we tried to define optimized inclusion and exclusion criteria but cannot totally preclude selection bias as we excluded not only patients with proven ischemic heart disease but also those with a mixed picture of ischemic/non-ischemic LGE. Moreover, our criteria might have been imprecise in a few cases leading to incorrect assignment or wrong exclusion of subjects: e.g. patients with end-stage HCM might also demonstrate thinning of LV wall with motion abnormality that is usually found in ischemic-damage LGE. Second, readers were blinded to all clinical information and were asked to focus on the late-enhancement sequences only, without taking into account other helpful images such as T2-weighted sequences to assess the presence of edema e.g. in inflammatory diseases ${ }^{[20]}$ or non-contrast SSFP images to judge cardiac function in cardiomyopathies. However, we believe this to be a limitation and strength at the same time, as we tried to avoid biases induced by clinical parameters or non-contrast sequences in order to completely focus on LGE pattern. Third, major parts of our study were descriptive, which might come along with two disadvantages: (1) results were influenced by the readers' subjective ratings and (2) results could only be qualitatively expressed, although quantitative results might have provided our study with a more informative character. Forth, although our study population comprised more individuals than the majority of other studies examining non-ischemic enhancement, certain diseases are underrepresented. Amyloidosis, for example, an important differential diagnosis was solely seen in 2 patients; thus, a reliable statement regarding LGE patterns indicative of this disease was not possible. Finally, comparison of our data with results published so far is hampered as pattern description in other studies is often confined to LGE localization within the LV wall, while information on its distribution, the LV wall preferentially affected and/or its signal intensity are only sporadically given. However, this underlines the uniqueness of our approach and the necessity to include different characteristics in pattern description.

In conclusion, LGE in cardiac MRI can be found in a variety of non-ischemic myocardial disorders and systematic analysis of the LGE pattern, as performed in the present study, might point to the right diagnosis. It will be the task of large future studies to assess whether detailed reading of the LGE pattern in conjunction with information gained from other sequences or clinical data will help to improve diagnostic reliability and specificity in the diagnosis of non-ischemic cardiac diseases, thereby influencing clinical management strategies.

\section{References}

[1] Cummings KW, Bhalla S, Javidan-Nejad C, Bierhals AJ, Gutierrez FR, Woodard PK. A pattern-based approach to assessment of delayed enhancement in nonischemic cardiomyopathy at MR imaging. Radiogr Rev Publ Radiol Soc North Am Inc. 2009; 29: 89-103.

[2] Hunold P, Schlosser T, Vogt FM, et al. Myocardial late enhancement in contrast-enhanced cardiac MRI: distinction between infarction scar and non-infarction-related disease. AJR Am J Roentgenol. 2005; 184: 1420-1426. http://dx.doi.org/10.2214/ajr.184.5.01841420

[3] Wu KC, Weiss RG, Thiemann DR, et al. Late gadolinium enhancement by cardiovascular magnetic resonance heralds an adverse prognosis in nonischemic cardiomyopathy. J Am Coll Cardiol. 2008; 51: 2414-2421. http://dx.doi.org/10.1016/j.jacc.2008.03.018

[4] Lim RP, Srichai MB, Lee VS. Non-ischemic causes of delayed myocardial hyperenhancement on MRI. AJR Am J Roentgenol. 2007; 188: 1675-1681. http://dx.doi.org/10.2214/AJR.06.1224 
[5] Bohl S, Wassmuth R, Abdel-Aty H, et al. Delayed enhancement cardiac magnetic resonance imaging reveals typical patterns of myocardial injury in patients with various forms of non-ischemic heart disease. Int J Cardiovasc Imaging. 2008; 24 : 597-607. http://dx.doi.org/10.1007/s10554-008-9300-x

[6] Choi E-Y, Choi BW, Kim S-A, et al. Patterns of late gadolinium enhancement are associated with ventricular stiffness in patients with advanced non-ischaemic dilated cardiomyopathy. Eur J Heart Fail. 2009; 11: 573-580. http://dx.doi.org/10.1093/eurjhf/hfp050

[7] Maceira AM, Joshi J, Prasad SK, et al. Cardiovascular magnetic resonance in cardiac amyloidosis. Circulation. 2005 ; $111: 186-193$. http://dx.doi.org/10.1161/01.CIR.0000152819.97857.9D

[8] Matoh F, Satoh H, Shiraki K, et al. The usefulness of delayed enhancement magnetic resonance imaging for diagnosis and evaluation of cardiac function in patients with cardiac sarcoidosis. J Cardiol. 2008; 51: 179-188. http://dx.doi.org/10.1016/j.jjcc.2008.03.002

[9] Nassenstein K, Bruder O, Breuckmann F, Erbel R, Barkhausen J, Schlosser T. Prevalence, pattern, and functional impact of late gadolinium enhancement in left ventricular hypertrophy due to aortic valve stenosis. RöFo Fortschritte Auf Dem Geb Röntgenstrahlen Nukl. 2009; 181: 472-476. http://dx.doi.org/10.1055/s-0028-1109044

[10] Vöhringer M, Mahrholdt H, Yilmaz A, Sechtem U. Significance of late gadolinium enhancement in cardiovascular magnetic resonance imaging (CMR). Herz. 2007; 32: 129-137. http://dx.doi.org/10.1007/s00059-007-2972-5

[11] Stork A, Müllerleile K, Bansmann PM, et al. [Patterns of delayed-enhancement in MRI of ischemic and non-ischemic cardiomyopathies]. RöFo Fortschritte Auf Dem Geb Röntgenstrahlen Nukl. 2007; 179: 21-30. http://dx.doi.org/10.1055/s-2006-927204

[12] Cerqueira MD, Weissman NJ, Dilsizian V, et al. Standardized myocardial segmentation and nomenclature for tomographic imaging of the heart. A statement for healthcare professionals from the Cardiac Imaging Committee of the Council on Clinical Cardiology of the American Heart Association. Int J Cardiovasc Imaging. 2002; 18: 539-542

[13] Kim RJ, Wu E, Rafael A, et al. The use of contrast-enhanced magnetic resonance imaging to identify reversible myocardial dysfunction. N Engl J Med. 2000; 343: 1445-1453. http://dx.doi.org/10.1056/NEJM200011163432003

[14] Simonetti OP, Kim RJ, Fieno DS, et al. An improved MR imaging technique for the visualization of myocardial infarction. Radiology. 2001; 218: 215-223. http://dx.doi.org/10.1148/radiology.218.1.r01ja50215

[15] Bruder O, Wagner A, Lombardi M, et al. European Cardiovascular Magnetic Resonance (EuroCMR) registry--multi national results from 57 centers in 15 countries. J Cardiovasc Magn Reson Off J Soc Cardiovasc Magn Reson. 2013; 15: 9.

[16] McCrohon JA, Moon JCC, Prasad SK, et al. Differentiation of heart failure related to dilated cardiomyopathy and coronary artery disease using gadolinium-enhanced cardiovascular magnetic resonance. Circulation. 2003; 108: 54-59.

http://dx.doi.org/10.1161/01.CIR.0000078641.19365.4C

[17] Maron MS. Clinical utility of cardiovascular magnetic resonance in hypertrophic cardiomyopathy. J Cardiovasc Magn Reson Off J Soc Cardiovasc Magn Reson. 2012; 14: 13

[18] Koito H, Suzuki J, Nakamori H, et al. [Clinical significance of abnormal high signal intensity of left ventricular myocardium by gadolinium-diethylenetriaminepenta-acetic acid enhanced magnetic resonance imaging in hypertrophic cardiomyopathy]. J Cardiol. 1995; 25: 163-170.

[19] Moon JCC, Sachdev B, Elkington AG, et al. Gadolinium enhanced cardiovascuAsease. Evidence for a disease specific abnormality of the myocardial interstitium. Eur Heart J. 2003; 24: 2151-2155. http://dx.doi.org/10.1016/j.ehj.2003.09.017

[20] Shimada T, Shimada K, Sakane T, et al. Diagnosis of cardiac sarcoidosis and evaluation of the effects of steroid therapy by gadolinium-DTPA-enhanced magnetic resonance imaging. Am J Med. 2001; 110: 520-527.

http://dx.doi.org/10.1016/S0002-9343(01)00677-5 